CREAT. MATH. INFORM.

Volume 29 (2020), No. 2,

Pages 131 - 136
Online version at https : //creative-mathematics. cunbm. utcluj.ro/

Print Edition: ISSN 1584 - 286X; Online Edition: ISSN 1843 - 441X

DOI: https://doi.org/10.37193/CMI.2020.02.03

\title{
On planarity of compressed zero-divisor graphs associated to commutative rings
}

\author{
M. ImRAN BHAT ${ }^{1}$, S. PiRZADA ${ }^{2}$ and AHMAD M. AlghAMDI ${ }^{3}$
}

\begin{abstract}
The equivalence class $[r]$ of an element $r \in \mathrm{R}$ is the set of zero-divisors $s$ such that ann $(r)=$ $\operatorname{ann}(s)$, that is, $[r]=\left\{s \in \mathrm{R}: \operatorname{ann}(r)=\operatorname{ann}(s)\right.$. The compressed zero-divisor graph, denoted by $\Gamma_{c}(\mathrm{R})$, is the compression of a zero-divisor graph, in which the vertex set is the set of all equivalence classes of nonzero zerodivisors of a ring $\mathrm{R}$, that is, the vertex set of $\Gamma_{c}(\mathrm{R})$ is $\mathrm{R}_{e}-\{[0],[1]\}$, where $\mathrm{R}_{e}=\{[r]: r \in \mathrm{R}\}$ and two distinct equivalence classes $[r]$ and $[s]$ are adjacent if and only if $r s=0$. In this article, we investigate the planarity of $\Gamma_{c}(\mathrm{R})$ for some finite local rings of order $p^{2}, p^{3}$ and determine the planarity of compressed zero-divisor graph of some local rings of order 32, whose zero-divisor graph is nonplanar. Further, we determine values of $m$ and $n$ for which $\Gamma_{c}\left(\mathbb{Z}_{n}\right)$ and $\Gamma_{c}\left(\mathbb{Z}_{n}[x] /\left(x^{m}\right)\right)$ are planar.
\end{abstract}

\section{INTRODUCTION}

In this article, $\mathrm{R}$ will denote a finite commutative ring with unity, unless otherwise stated, $Z^{\star}(\mathrm{R})=Z(\mathrm{R}) \backslash\{0\}$ denotes the set of all nonzero zero-divisors of $\mathrm{R}$. We will denote a finite field on $q$ of elements by $\mathcal{F}_{q}$, ring of integers modulo $n$ by $\mathbb{Z}_{n}$. A ring $\mathrm{R}$ is said to be a local ring if and only if $\mathrm{R}$ has a unique maximal ideal. An element $x \in \mathrm{R}$ is nilpotent if $x^{n}=0$ for some $n \in \mathbb{N}$. A ring $\mathrm{R}$ is a reduced ring if it contains no nonzero nilpotent element. An annihilator of an element $x$ of a ring $\mathrm{R}$ is defined as $\operatorname{ann}(x)=\{r \in \mathrm{R} \mid r x=0\}$.

We consider a simple graph $\mathcal{G}(\mathcal{V}, \mathcal{E})$ with vertex set $\mathcal{V}$ and edge set $\mathcal{E}$. A path between two vertices $x_{1}, x_{n} \in \mathcal{V}(\mathcal{G})$ is an ordered sequence of distinct vertices $x_{1}, x_{2}, \ldots, x_{n}$ of $\mathcal{G}$ such that $x_{i-1} x_{i}$ is an edge for $2 \leq i \leq n$. A graph $\mathcal{G}$ is connected if and only if there exists a path between every pair of vertices in $\mathcal{G}$. The distance between two vertices $x$ and $y$, denoted by $d(x, y)$, is the length of the shortest $x-y$ path and the diameter of a graph $\mathcal{G}$ is $\operatorname{diam}(\mathcal{G})=\sup \{d(x, y) \mid x$ and $y$ are distinct vertices of $\mathcal{G}\}$. We denote a complete graph with $n$ vertices by $\mathcal{K}_{n}$ and the complete bipartite graph with $m$ and $n$ vertices in two parts by $\mathcal{K}_{m, n}$. A graph is regular if each of its vertex has the same degree. A graph $\mathcal{G}$ is said to be planar if there exists a drawing of $\mathcal{G}$ in the plane in which no two edges intersect in a point other than a vertex of $\mathcal{G}$. Such a drawing of a planar graph is called planar representation of $\mathcal{G}$. For undefined terms and notations, we refer to $[5,18]$.

The study of linking graph theory with commutative ring theory was first given by Beck [6] in 1988. Anderson and Livingston [2] modified the definition and studied the zero-divisor graph $\Gamma(\mathrm{R})$ whose vertex set is the set of nonzero zero-divisors, denoted by $Z^{\star}(\mathrm{R})=Z(\mathrm{R})-\{0\}$ of the commutative ring $\mathrm{R}$ and the two distinct vertices $x$ and $y$ are adjacent if and only if $x y=0$.

The compressed zero-divisor graph, denoted by $\Gamma_{c}(\mathrm{R})$, is the compression of a zerodivisor graph, in which the vertex set is the set of all equivalence classes of nonzero zerodivisors of a ring $\mathrm{R}$, that is, the vertex set of $\Gamma_{c}(\mathrm{R})$ is $Z\left(\mathrm{R}_{c}\right) \backslash\{[0]\}=\mathrm{R}_{c} \backslash\{[0],[1]\}$ defined by $\mathrm{R}_{c}=\{[r]: r \in \mathrm{R}\}$, where $[r]=\{s \in \mathrm{R}: \operatorname{ann}(r)=$ ann $(s)\}$ and two distinct equivalence

Received: 17.05.2020. In revised form: 08.09.2020. Accepted: 15.09.2020

2010 Mathematics Subject Classification. 13A99, 05 C78.

Key words and phrases. Zero-divisor graph, compressed zero-divisor graph, planarity, local ring.

Corresponding author: S. Pirzada; pirzadasd@kashmiruniversity.ac.in 
classes $[r]$ and $[s]$ are adjacent if and only if $r s=0$. This compressed zero-divisor graph $\Gamma_{c}(R)$ can be a finite graph even if $R$ is an infinite ring and $\Gamma(R)$ is an infinite graph. More on compressed zero-divisor graphs can be seen in $[3,10,19,21]$.

Anderson et al. [1] determined all $n$ for which $\Gamma\left(\mathbb{Z}_{n}\right)$ is planar, and posed the question of which finite rings in general determine a planar zero-divisor graph. This was answered by Akbari et al [4], who refined the question to local rings of cardinality at most 32. Belshoff and Chapman [7] proved that if $R$ is any local ring with more than 27 elements, and $R$ is not a field, then $\Gamma(R)$ is non planar.

The following result is useful for further reference in this paper.

Theorem 1.1. (Kuratowski) A graph is planar if and only if it no contains subdivision homeomorphic to $\mathcal{K}_{5}$ or $\mathcal{K}_{3,3}$.

The concept of metric dimension of graphs was introduced by Harary and Melter [11] in the following way: Let $\mathcal{G}$ be a connected graph of order $n \geq 1$. If $\mathcal{M}_{\mathcal{B}}=\left\{w_{1}, w_{2}, \ldots, w_{k}\right\}$ is an (ordered) set of vertices, the metric vector of a vertex $v \in \mathcal{G}$ relative to $\mathcal{M}_{\mathcal{B}}$ is the vector $r_{\mathcal{M}_{\mathcal{B}}}(v)=\left(d\left(v, w_{1}\right), d\left(v, w_{2}\right), \ldots, d\left(v, w_{k}\right)\right)$. The set $\mathcal{M}_{\mathcal{B}}$ is a resolving set if distinct vertices have distinct metric vectors and a minimum resolving set is called a metric basis for $\mathcal{G}$ and its cardinality, denoted by $\mathcal{M}_{d}(G)$, is called the metric dimension of $\mathcal{G}$. Recent work on metric and upper dimension on graphs associated to rings can be seen in $[8,13,14,15,16,17,20]$.

Theorem 1.2. [Theorem 3.2, [12]] A graph with metric dimension 2 can not have $\mathcal{K}_{3,3}$ or $\mathcal{K}_{5}$ as a subgraph.

In this paper, we will investigate the planarity of compressed zero-divisor graph associated to commutative rings.

\section{Planarity of compressed zero-divisor graphs}

The planarity of a zero-divisor graph implies the planarity of the corresponding compressed zero-divisor graph as can be seen below.

Theorem 2.3. If $\Gamma(\mathrm{R})$ is planar, then $\Gamma_{c}(\mathrm{R})$ is also planar.

Proof. Let the zero-divisor graph $\Gamma(\mathrm{R})$ associated to a ring $\mathrm{R}$ be planar. That is, there is no crossover between the edges of the graph $\Gamma(R)$. We know that the vertices of $\Gamma_{c}(R)$ are the equivalence classes (annihilators) of zero-divisors in $\mathcal{Z}^{\star}(\mathrm{R})$. Let $a$ and $\mathrm{b}$ be two distinct vertices in $\Gamma(\mathrm{R})$. If $\operatorname{ann}(\mathrm{a}) \neq \operatorname{ann}(\mathrm{b})$, then $[\mathrm{a}]$ and $[\mathrm{b}]$ are the vertices in $\Gamma_{c}(\mathrm{R})$, otherwise collapse to a single vertex. Thus, both the order as well as size of the graph $\Gamma_{c}(R)$ is either smaller or equal to the order and size of $\Gamma(R)$. That is, $\Gamma_{c}(R)$ is a subgraph of $\Gamma(R)$. Since subgraph of a planar graph is planar, it follows that $\Gamma_{c}(\mathrm{R})$ is planar (see example, Fig. 2(a)).

The converse of Theorem 2.3 is not true in general. If $\Gamma(R)$ is nonplanar, then $\Gamma_{c}(R)$ may be planar(Figure1), except the case, when $R \cong\left(\Pi_{i=1}^{n} \mathbb{Z}_{2}\right)$, in which case $\Gamma(R) \cong \Gamma_{c}(R)$. See Figure 2(b).

Theorem 2.4. If $\mathrm{R} \cong \mathcal{F}_{\mathrm{p}} \times \mathcal{F}_{\mathrm{q}}$, where $\mathcal{F}_{\mathrm{p}}$ and $\mathcal{F}_{\mathrm{q}}$ are prime fields, then $\Gamma_{c}(\mathrm{R})$ is planar.

Proof. Let $\mathrm{R} \cong \mathcal{F}_{\mathrm{p}} \times \mathcal{F}_{\mathrm{q}}$. Then there exist two maximal ideals $\mathcal{F}_{\mathrm{p}} \times 0$ and $F_{\mathrm{q}} \times 0$, which are the only maximal ideals in $\mathrm{R}$, so all other ideals (and therefore all other annihilator sets) are subsets of either $F_{\mathrm{p}} \times 0$ or $F_{\mathrm{q}} \times 0$. We see that $\operatorname{ann}((0,1))=(a, 0) \mid a \in F_{p}$ and 
$\operatorname{ann}((1,0))=(0, b) \mid b \in F_{q}$. Moreover, it is easy to see that $\operatorname{ann}((0,1))-\operatorname{ann}((1,0))=(0,0)$. Since we do not allow units in the vertex set of a zero-divisor graph, there is no vertex whose annihilator set is a subset of both maximal ideals. Thus, $\left|\mathcal{Z}^{\star}\left(F_{\mathrm{p}} \times F_{\mathrm{q}}\right)_{c}\right|=2$, that is, $\Gamma_{c}(\mathrm{R})$ is a single edge.

Theorem 2.5. If $\mathrm{R}$ is a finite commutative ring with unity 1 such that $\mathrm{R} \cong \mathrm{R}_{1} \times \mathrm{R}_{2}$, where $\mathrm{R}_{1}$ and $\mathrm{R}_{2}$ are local rings, then $\Gamma_{c}(\mathrm{R})$ is planar if at least one $\mathrm{R}_{\mathrm{i}}$ is a field.

Proof. Let $\mathrm{R}$ be a finite commutative ring with unity 1 such that $R \cong R_{1} \times R_{2}$, where $R_{1}$ and $R_{2}$ are local rings. Therefore, the equivalence classes of $\Gamma_{c}\left(R_{1} \times R_{2}\right)$ are of the form $[(0,1)]$, $[(1,0)],[(a, 0)],[(0, b)],[(a, 1)],[(1, b)],[(a, b)]$, where $a$ and $b$ are the nonzero zero-divisors in $R_{1}$ and $R_{2}$ respectively. If $R_{1}$ or $R_{2}$ is a field, then the edges of $\Gamma_{c}(R)$ are $[(0,1)][(1,0)]$, $[(0,1)][(a, 0)],[(1,0)][(0, b)]$. Clearly, the graph $\Gamma_{c}(\mathrm{R})$ contains no induced subgraph $\mathcal{K}_{5}$ or $\mathcal{K}_{3,3}$. Hence $\Gamma_{c}(\mathrm{R})$ is planar.

A maximal complete subgraph of $\mathcal{G}$ is a clique of $\mathcal{G}$. The order of a maximal clique of $\mathcal{G}$ is the clique number, denoted by $\operatorname{cl} q(\mathcal{G})$.

Theorem 2.6. Let $\mathrm{p}$ be a prime number and $\mathrm{n} \in \mathbb{N}$. Then $\Gamma_{c}\left(\mathbb{Z}_{\mathrm{p}^{\mathrm{n}}}\right)$ is planar if and only if $\mathrm{n} \leq 8$.

Proof. When $n>3$ and $p \geq 2$, we partition the vertex set of $\Gamma\left(\mathbb{Z}_{p^{n}}\right)$ into disjoint subsets $\mathcal{V}_{1}, \mathcal{V}_{2}, \cdots, \mathcal{V}_{n-1}$, where $\mathcal{V}_{i}=\left\{k_{i} p^{i}: p \nmid k_{i}\right\}, 1 \leq i \leq n-1$. Then it is easy to see that $\left|V_{i}\right|=(p-1) p^{n-i-1}, 1 \leq i \leq n-1$ and therefore $\left|\Gamma\left(\mathbb{Z}_{p^{n}}\right)\right|=\sum_{i=1}^{n-1}(p-1) p^{n-i-1}=p^{n-1}-1$.

Now the equivalence classes of zero-divisors of $\mathbb{Z}_{p^{n}}$ are

$$
[u p]=\left\{u p^{n-1}\right\},\left[u p^{2}\right]=\left\{u p^{n-1}, u p^{n-2}\right\}, \ldots,\left[u p^{n-1}\right]=\left\{u p^{n-1}, u p^{n-2}, \ldots, u p^{2}, u p\right\} .
$$

In this way, we get $n-1$ distinct equivalence classes. Therefore, $\left|\Gamma_{c}\left(\mathbb{Z}_{n}\right)\right|=n-1$.

Using this partitioning, we see that $v_{i}$ is adjacent to $v_{j}$ for each $\left[\frac{n}{2}\right] \leq i, j \leq n-1$. Thus, the clique number of $\Gamma_{c}\left(\mathbb{Z}_{\mathrm{p}^{n}}\right)$ is given by

$$
\operatorname{clq}\left(\Gamma_{c}\left(\mathbb{Z}_{\mathrm{p}^{\mathrm{n}}}\right)\right)(\mathrm{n}-1)-\left(\left[\frac{n}{2}\right]-1\right)= \begin{cases}\frac{\mathrm{n}}{2}, & \text { if } n \text { is even } \\ \frac{\mathrm{n}+1}{2}, & \text { if } n \text { is odd } .\end{cases}
$$

Using this formula, for $\mathrm{n} \leq 8$, it is clear that $\Gamma_{c}\left(\mathbb{Z}_{\mathrm{p}^{\mathrm{n}}}\right)$ is planar. If $\mathrm{n} \geq 9$, then $\operatorname{cl}\left(\Gamma_{c}\left(\mathbb{Z}_{\mathrm{p}^{\mathrm{n}}}\right)\right) \geq$ 5 , that is, $\Gamma_{c}\left(\mathbb{Z}_{\mathrm{p}^{\mathrm{n}}}\right)$ contains a maximal clique $\mathcal{K}_{5}$ and hence is nonplanar.

Theorem 2.7. Let $\mathrm{R}$ be a finite commutative reduced ring, that is, $\mathrm{R} \cong \mathcal{F}_{1} \times \mathcal{F}_{2} \times \cdots \times \mathcal{F}_{\mathrm{n}}$. Then, $\Gamma_{c}(\mathrm{R})$ is planar only if $n \leq 3$.

Proof. If $R$ is not a reduced ring, then either $R$ is a local ring or $R \cong R_{1} \times R_{2} \times \ldots R_{n}$, where at least one $R_{i}(1 \leq i \leq n)$ is not a field. So, if $R$ is a reduced ring, then $R \cong \mathcal{F}_{1} \times \mathcal{F}_{2} \times \cdots \times \mathcal{F}_{n}$ for some finite fields $\mathcal{F}_{\mathrm{i}}(1 \leq \mathrm{i} \leq \mathrm{n})$.

If $\mathrm{n}=2$, that is, $\mathrm{R} \cong \mathcal{F}_{1} \times \mathcal{F}_{2}$, then by Corollary 2.4, $\Gamma_{c}(\mathrm{R}) \cong \mathcal{K}_{2}$ which is planar.

If $\mathrm{n}=3$, then $\mathrm{R} \cong \mathcal{F}_{1} \times \mathcal{F}_{2} \times \mathcal{F}_{3}$ gives six different equivalence classes; $[(1,1,0)],[(1,0,1)],[(0,1,1)],[(1,0,0)],[(0,1,0)],[(0,0,1)]$ as vertices of $\Gamma_{c}(R)$. The edges between the equivalence classes $[(1,0,0)],[(0,1,0)],[(0,0,1)]$ always form a maximal clique with each vertex of this clique has a pendent edge. Also, the metric dimension of this graph is 2 and the result follows by Theorem 1.2. Thus, in this case $\Gamma_{c}(R)$ is planar.

If $\mathrm{n} \geq 4$, then $\Gamma_{c}(\mathrm{R})$ is nonplanar, for example, consider the ring $\mathrm{R} \cong\left(\Pi_{\mathrm{i}=1}^{n} \mathcal{Z}_{2}\right), \mathrm{n}=4$. Its $\Gamma(\mathrm{R}) \cong \Gamma_{c}(\mathrm{R})$, whenever $\mathrm{R}$ is a Boolean ring. 
Theorem 2.7 can be proved directly by using the definition itself. In that case, we need to investigate the structure of the graph which becomes complicated for large graphs. The proof given above is simple.

Theorem 2.8. Let $\mathrm{R}$ be a commutative ring with unity $1 \neq 0$, (not a domain). If $\mathrm{R}$ is a local ring of order $\mathrm{p}^{2}, \mathrm{p}^{3}$ and 16 , then $\Gamma_{c}(\mathrm{R})$ is planar.

Proof. If $\mathrm{R}$ is local ring of order $\mathrm{p}^{2}$, then either $\mathrm{R}$ is isomorphic to $\mathbb{Z}_{p^{2}}$ or $\frac{\mathbb{Z}_{p}[x]}{x^{2}}$ and in either case $\Gamma(\mathrm{R})$ has order $p-1$, which then by definition of compressed zero-divisor graph collapses to a single vertex. Note that, if $\mathrm{R}$ is reduced, then $\mathrm{R}$ isomorphic to $\mathbb{Z}_{p} \times \mathbb{Z}_{p}$, so $\Gamma_{c}(\mathrm{R}) \cong \mathcal{K}_{1,1}$, by [Proposition 2, [19]]. Corbas et al. [9] characterized all local ring of order $\mathrm{p}^{3}$, given as; $\mathbb{Z}_{p^{3}}, \frac{\mathbb{Z}_{p}[x]}{\left(x^{3}\right)}, \frac{\mathbb{F}_{p}[x, y]}{(x, y)^{2}}, \frac{\mathbb{Z}_{p^{2}}[x]}{\left(\epsilon x, x^{2}\right)} \frac{\mathbb{Z}_{p^{2}}[x]}{\left(p x, x^{2}-\epsilon x\right)}$, where $\epsilon \in \mathbb{Z}_{p}$ is a nonsquare element. Also, the compressed zero-divisor graph of the rings of order 16 have metric dimension at most 2 . In each case, $\Gamma_{c}(\mathrm{R})$ is planar, follows from [Proposition 2, 4 and 5, [19]].

The following result shows that $\Gamma_{c}(\mathrm{R})$ is planar, when $\mathrm{R}$ is a local ring of order 32.

Theorem 2.9. If $\mathrm{R}$ is a finite local ring of order 32 , then $\Gamma_{c}(\mathrm{R})$ is planar.

Proof. The various commutative local rings of order 32 are as follows.

(1) $\mathrm{R}=\mathbb{F}_{32}$, then $\Gamma_{c}(\mathrm{R})$ is an empty graph.

(2) $\mathrm{R}=\frac{\mathbb{Z}_{2}[x]}{\left(x^{5}\right)}$. Determining the equivalence classes of zero-divisors of this ring, the vertices of $\Gamma_{c}(\mathrm{R})$ are $u_{1}=[x], u_{2}=\left[x^{2}\right], u_{3}=\left[x^{3}\right], u_{4}=\left[x^{4}\right]$. It is to see that $u_{i} u_{j}=0$ for all $i, j$ and clearly, the graph $\Gamma_{c}(\mathrm{R})$ on these vertices is planar as shown in Fig.3(a).

(3) $\mathrm{R}=\frac{\mathbb{Z}_{2}[x, y]}{\left(x^{4}, x y, y^{2}-x^{3}\right)}$. The zero-divisor set of this ring is $\mathrm{Z}(\mathrm{R})=\left\{0, x, y, x^{2}, y^{2}, x+\right.$ $y, x+y^{2}, y+x^{2}, y+y^{2}, x^{2}+y^{2}, x+y+y^{2}, x+y+x^{2}, x+x^{2}+y^{2}, y+x^{2}+$ $\left.y^{2}, x+y+x^{2}+y^{2}\right\}$. The set of annihilator ideals of these zero-divisors is $\mathrm{Z}(\mathrm{R})_{c}=$ $\left\{[x],[y],\left[x^{2}\right],\left[y^{2}\right],[x+y],\left[x^{2}+y\right],\left[y+y^{2}\right]\right\}$. Clearly, $\Gamma_{c}(\mathrm{R})$ is a planar graph shown in Fig.3(b).

(4) $\mathrm{R}=\frac{Z_{2}[x, y]}{\left(x^{4}, x y, y^{2}\right)}, \mathrm{Z}(\mathrm{R})=\left\{0, x, y, x+y, x^{2}, x^{3}, x+x^{2}, y+x^{2}, x+x^{3}, y+x^{3}, x^{2}+\right.$ $\left.x^{3}, x+y+x^{2}, x+y+x^{3}, x+x^{2}+x^{3}, y+x^{2}+x^{3}, x+y+x^{2}+x^{3}\right\}$. So that, $\mathrm{Z}(\mathrm{R})_{c}=\left\{[x],\left[x^{2}\right],[y]\right\}$. In this case $\Gamma_{c}(\mathrm{R})$ is shown in Fig.3(c), which is obviously planar.

(5) $\mathrm{R}=\frac{Z_{4}[x]}{\left(2 x, x^{4}-2\right)}, \mathrm{Z}(\mathrm{R})=\left\{0,2, x, x^{2}, x^{3}, x+x^{2}, x+x^{3}, x+2, x^{2}+2, x^{2}+x^{3}, x+\right.$ $\left.x^{2}+2, x^{3}+2, x+x^{3}+2, x+x^{2}+x^{3}, x^{2}+x^{3}+2, x+x^{2}+x^{3}+2\right\}$, Then the set of vertices of $\Gamma_{c}(\mathrm{R})$ is $\mathrm{Z}(\mathrm{R})_{c}=\left\{[2],[x],\left[x^{2}\right],\left[x^{3}\right]\right\}$. Fig.3(d), shows that $\Gamma_{c}(\mathrm{R})$ is planar.

(6) $\mathrm{R}=\frac{\mathbb{Z}_{4}[x]}{\left(2 x^{2}, x^{3}-2\right)}, \mathrm{Z}(\mathrm{R})=\left\{0,2, x, x^{2}, x+2, x^{2}+2,2 x, 3 x, 2 x+2,3 x+2, x+x^{2}, 2 x+\right.$ $\left.x^{2}, 3 x+x^{2}, x+x^{2}+2,2 x+x^{2}+2,3 x+x^{2}+2\right\}$. Then $\mathrm{Z}(\mathrm{R})_{c}=\left\{[x],[2],[2 x],\left[x^{2}\right]\right\}$. Therefore, $\Gamma_{c}(\mathrm{R})$ is planar as shown in Fig.3(e).

It may be recalled here that the genus $\gamma$ of a graph $\mathcal{G}$ is the minimum number of handles which must be added to a sphere so that $\mathcal{G}$ can be embedded on the resulting surface. Clearly, $\gamma(\mathrm{G})=0$ if and only if $\mathcal{G}$ is planar. 
Analogous to some results in [22], we have the following.

Theorem 2.10. Let $\mathrm{R}$ be a homomorphic image of $\mathbb{Z}^{\mathrm{n}}$. Then $\Gamma_{c}(\mathrm{R})$ is planar if and only if $\mathrm{R}$ is one of the rings $\mathbb{Z}_{\mathrm{p}^{n}}(\mathrm{n} \leq 8), \mathbb{Z}_{\mathrm{p}} \times \mathbb{Z}_{\mathrm{q}}, \mathbb{Z}_{\mathrm{p}} \times \mathbb{Z}_{\mathrm{p}}, \mathbb{Z}_{\mathrm{p}^{2}} \times \mathbb{Z}_{\mathrm{q}}, \mathbb{Z}_{4} \times \mathbb{Z}_{4}, \mathbb{Z}_{4} \times \mathbb{Z}_{9}, \mathbb{Z}_{\mathrm{p}^{3}} \times \mathbb{Z}_{\mathrm{q}_{\mathrm{q}}}, \mathbb{Z}_{\mathrm{p}^{3}} \times \mathbb{Z}_{\mathrm{q}}$, $\mathbb{Z}_{\mathrm{p}_{1}} \times \mathbb{Z}_{\mathrm{p}_{2}} \times \mathbb{Z}_{\mathrm{p}_{3}}, \mathbb{Z}_{2} \times \mathbb{Z}_{2} \times \mathbb{Z}_{4}$.

Proof. We prove the result by factorizing $\mathrm{n}$.

(1) If $\mathrm{n}=\mathrm{p}$, then $\Gamma_{c}(\mathrm{R})$ is planar as $\Gamma_{c}\left(\mathbb{Z}_{\mathrm{p}}\right)=\phi$.

(2) If $\mathrm{n}=\mathrm{p}^{\mathrm{n}}$, then by Theorem $2.6 ; \Gamma_{c}\left(\mathbb{Z}_{\mathrm{p}^{\mathrm{n}}}\right)$ does not contain any $\mathcal{K}_{3,3}$ or $\mathcal{K}_{5}$ as a subgraph, because the clique number is less than 5 for $n \leq 8$. Therefore, $\Gamma_{c}\left(\mathbb{Z}_{\mathrm{p}^{n}}\right)$ is planar for $\mathrm{n} \leq 8$. Moreover, if $\mathrm{n} \geq 9$, then $\Gamma_{c}\left(\mathbb{Z}_{\mathrm{p}^{\mathrm{n}}}\right)$ is nonplanar, follows from Theorem 2.6.

(3) If $\mathrm{n}=\mathrm{p} \times \mathrm{q}$, then $\Gamma_{c}\left(\mathbb{Z}_{\mathrm{p}} \times \mathbb{Z}_{\mathrm{q}}\right)$ is planar, follows from Corollary 2.4.

(4) If $\mathrm{n}=\mathrm{p} \times \mathrm{p}$, then $\Gamma_{c}\left(\mathbb{Z}_{\mathrm{p}} \times \mathbb{Z}_{\mathrm{p}}\right)$ is planar, follows from Corollary 2.4.

(5) If $\mathrm{n}=\mathrm{p}^{2} \times \mathrm{q}$, then $\Gamma_{c}\left(\mathbb{Z}_{\mathrm{p}^{2} \times \mathbb{Z}_{\mathrm{q}}}\right)$ is planar, follows from Theorem 2.5 and Corollary 2.4.

(6) If $\mathrm{n}=\mathrm{p}^{2} \times \mathrm{q}^{2}$, then $\Gamma_{c}\left(\mathbb{Z}_{4} \times \mathbb{Z}_{4}\right)$ and $\Gamma_{c}\left(\mathbb{Z}_{4} \times \mathbb{Z}_{9}\right)$ are planar. Moreover, if $\mathrm{p}=\mathrm{q} \geq 3$, then $\Gamma_{c}\left(\mathbb{Z}_{\mathrm{p}^{2}} \times \mathbb{Z}_{\mathrm{q}^{2}}\right)$ is nonplanar. Thus, $\Gamma_{c}\left(\mathbb{Z}_{\mathrm{p}^{2}} \times \mathbb{Z}_{\mathrm{q}^{2}}\right)$ is nonplanar for distinct primes $\mathrm{p} \geq 3$ and $q \geq 3$.

(7) If $\mathrm{n}=\mathrm{p}^{3} \times \mathrm{q}$, then $\left|\mathcal{Z}^{\star}\left(\mathrm{R}_{c}\right)\right|=2(1+2)=6$. Then, $\Gamma_{c}\left(\mathbb{Z}_{\mathrm{p}^{3}} \times \mathbb{Z}_{\mathrm{q}}\right)$ is planar.

(8) Let $\mathrm{n}=\mathrm{p}^{3} \times \mathrm{q}^{\mathrm{k}}$. If $\mathrm{q}=2, \mathrm{k}=1$, it reduces to the case (7). $\Gamma_{c}(\mathrm{R})$ is nonplanar for $\mathrm{k} \geq 2$, as the graph contains $\mathcal{K}_{3,3}$ as a subgraph. Thus, $\Gamma_{c}\left(\mathbb{Z}_{\mathrm{p}^{3}} \times \mathbb{Z}_{\mathrm{q}^{\mathrm{k}}}\right)$ is planar if and only if $\mathrm{k}=1$.

(9) Let $n=p^{k} \times q^{t}$. If $k=1, t=1$, then it reduces to the case (3). If $k=2, t=2$, then the case reduces to (6). If $k \geq 2, t=1$, then the case reduces to (5). If $k=3, t \geq 2$, it reduces to (8). If $k \geq 3, t \geq 2$ and $p, q$ are distinct primes, then $\Gamma_{c}\left(\mathbb{Z}_{\mathrm{p}^{k}} \times \mathbb{Z}_{\mathrm{q}^{\mathrm{t}}}\right)$ is nonplanar.

(10) Let $\mathrm{n}=\mathrm{p}_{1} \times \mathrm{p}_{2} \times \mathrm{p}_{3}$. If each $\mathrm{p}_{\mathrm{i}}$ is a prime field, then it is easy to see that, $\Gamma_{c}\left(\mathbb{Z}_{\mathrm{p}_{1}} \times\right.$ $\left.\mathbb{Z}_{\mathrm{p}_{2}} \times \mathbb{Z}_{\mathrm{p}_{3}}\right)$ is planar. Also, $\Gamma_{c}\left(\mathbb{Z}_{2} \times \mathbb{Z}_{2} \times \mathbb{Z}_{4}\right)$ and $\Gamma_{c}\left(\mathbb{Z}_{2} \times \mathbb{Z}_{2} \times \mathbb{Z}_{6}\right)$ are planar. Moreover, $\Gamma_{c}\left(\mathbb{Z}_{2} \times \mathbb{Z}_{3} \times \mathbb{Z}_{4}\right)$ is nonplanar.

Now, we investigate the planarity of the compressed zero-divisor graph associated to the ring $\mathbb{Z}_{n}[X] /\left(X^{m}\right)$ for different integers $m$ and $n$.

Theorem 2.11. Let $\mathrm{R}_{m, n}=\mathbb{Z}_{n}[X] /\left(X^{m}\right)$, where $m, n \geq 2$. Then $\Gamma_{c}\left(\mathrm{R}_{m, n}\right)$ is planar for any one of the following. (i) $n=p q$ and $m \geq 2$ and $p>q$, (ii) $n=p^{2}$ and $m \geq 3$, (iii) $n=p$, (iv) $m \geq 6$.

Proof. (i) Let $\mathrm{R}=\mathbb{Z}_{p q}[X] /\left(X^{m}\right)$. Then $\mathrm{R} \cong \mathbb{Z}_{p} \times \mathbb{Z}_{q}$. Let $x=X+\left(X^{m}\right)$ be the image of $X$ in $\mathbb{Z}_{p} q[X] /\left(X^{m}\right)$. Since we define a relation on the set of zero-divisors with two zero-divisors $x, y \in Z^{*}(R)$ being equivalent if $\operatorname{ann}(x)=\operatorname{ann}(y)$, it is easy to see that the annihilators (equivalence classes) obtained from the zero-divisors of $\mathrm{R}$ which later act as the vertices of the compressed zero-divisor graph $\Gamma_{c}\left(\mathbb{Z}_{R}\right)$ are $u_{1}=[p]$ and $v_{1}=[q]$. Therefore, $\gamma=0$.

(ii) The zero-divisor set of the ring $\mathbb{Z}_{p^{2}}[X] /\left(X^{m}\right)$ is $Z(\mathrm{R})=\left\{p, p x^{m-1}, p x^{m-2}, p+x^{2}, p x^{m-1}+\right.$ $\left.p x^{m-2}, p+2 x^{2}, p+3 x^{2}, p+p x, p+p x+x^{2}, p+p x+2 x^{2}, p+p x+3 x^{2}\right\}$, so the vertices of $\Gamma_{c}\left(\mathrm{R}_{p}^{2}\right)$ are $\left[p x^{m-1}\right],[p]$.

(iii) Let $\mathrm{R}_{m, n}=\mathbb{Z}_{n}[X] /\left(X^{m}\right)$. If $n=p$, then the map $\left(a_{1}+a_{2} x+a_{n-1} x^{n-1}+a_{n} x^{n}\right) \mapsto$ $a_{1}+a_{2} p+a_{n-} p^{n-1}$ is an isomorphism between $\frac{\mathbb{F}_{p}[x]}{x^{n}}$ and $\mathbb{Z}_{p^{n}}$ and the planarity follows by Theorem 2.6.

(iv) If $m=4, n \geq 4$, the equivalence classes of each zero-divisor is same, that is, the zerodivisor graph turns out to be a single vertex. The compressed zero-divisor graph of a ring $\mathrm{R}_{m, n}=\mathbb{Z}_{n}[X] /\left(X^{m}\right), m \geq 6$, is planar, follows from Part (iii).

Acknowledgements. The authors are grateful to the anonymous referee for his useful suggestions. 
Imran, Pirzada, Alghamdi

\section{REFERENCES}

[1] Anderson, D. F., Frazier, A., Lauve, A. and Livingston, P. S., The zero-divisor graph of a commutative ring, II, Lecture Notes in Pure and Appl. Math., Marcel Dekker, New York, 220 (2001), 61-72

[2] Anderson, D. F. and Livingston, P. S., The zero-divisor graph of a commutative ring, J. Algebra 217 (1999), No. 2, 434-447

[3] Anderson, D. F. and LaGrange, J. D., Some remarks on the compressed zero-divisor graph, J. Algebra, 447 (2016), 297-321

[4] Akbari, S., Maimani, H. R. and Yassemi, S., When a zero-divisor graph is planar or a complete r-partite graph, J. Algebra, 270 (2003), No. 1, 169-180

[5] Atiyah, M. F. and Macdonald, I. G., Introduction to Commutative Algebra, Addison-Wesley Publishing, Reading, MA, (1969)

[6] Beck, I., Coloring of commutative rings, J. Algebra, 116 (1988), No. 1, 208-226

[7] Belshoff, R. and Chapman, J., Planar zero-divisor graphs. J. Algebra, 316 (2007), No. 1, 471-480

[8] Bhat, M. I. and Pirzada, S., On strong metric dimension of zero divisor graphs of rings, The Korean J. Mathematics, 27 (2019), No. 3, 563-580

[9] Corbas, B. and Williams, G. D., Rings of order $p^{5}$, II. Local rings, J. Algebra, 231 (2000), No. 2, 691-195

[10] Hashemi, E., Abdi, M. and Alhevaz, A., On the diameter of the compressed zero-divisor graph, Comm. Algebra, 45 (2017), No. 11, 4855-4864

[11] Harary, F. and Melter, R. A., On the metric dimension of a graph. Ars Combin., 2 (1976), 191-195

[12] Khuller, S., Raghavachari, B. and Rosenfield, A., Landmarks in graphs. Disc. Appl. Math., 70 (1996), No. 3 , 217-229

[13] Pirzada, S. Aijaz, M. and Redmond, S. P., Upper dimension and bases of zero divisor graphs of commutative rings, AKCE International J. Graphs Comb. DOI: 10.1016/j.akcej.2018.12.001

[14] Pirzada, S., Raja, R. and Redmond, S. P., Locating sets and numbers of graphs associated to commutative rings, J. Algebra Appl., 13 (2014), No. 7, 1450047 (18 pp.)

[15] Pirzada, S. and Aijaz, M., Metric and upper dimension of zero divisor graphs associated to commutative rings, Acta Univ. Sapientiae, Informatica, 12 (2020), No. 1, 84-101

[16] Pirzada, S. and Raja, R., On the metric dimension of a zero divisor graph, Communications Algebra, 45 (2017), No. 4, 1399-1408

[17] Pirzada, S., Aijaz, M. and Redmond, S. P., On upper dimension of graphs and their bases sets, Discrete Math. Letters, 3 (2020), 37-43

[18] Pirzada, S., An Introduction to Graph Theory. Universities Press, Hyderabad, India, (2012).

[19] Pirzada, S. and Bhat, M. I., Computing metric dimension of compressed zero-divisor graphs associated to rings, Acta Univ. Sapientiae, Mathematica, 10 (2018), No. 2, 298-318

[20] Raja, R., Pirzada, S. and Redmond, S. P., On locating numbers and codes of zero divisor graphs associated with commutative rings, J. Algebra Applications, 15 (2016), No. 1, 1650014 (22 pages)

[21] Spiroff, S. and Wickham, C., A zero divisor graph determined by equivalence classes of zero divisors, Comm. Algebra, 39 (2011), No. 7, 2338-2348

[22] Wang, H. J., Zero-divisor graphs of genus one, J. Algebra, 304 (2006), No. 2, 666-678

1,2 Department of MATHEMATiCs

UNIVERSITY OF KASHMIR

HAZRATBAL, SRINAGAR, KASHMIR, INDIA

Email address: imran.scholar@kashmiruniversity.net; pirzadasd@kashmiruniversity.ac.in

${ }^{3}$ Department of Mathematical SCIENCES

UMM ALQURA UNIVERSITY

SAUDI ARABIA

Email address: amghamdi@uqu. edu.sa 